\title{
Supporting Information \\ A Combined Relativistic Ab Initio Multireference and Experimental Study of the Electronic Structure of Terbium Luminescent Compound
}

\author{
Chun-Xiang Wang, ${ }^{\mathrm{a}, \dagger}$ Yong Li, ${ }^{*, \mathrm{~b}, \dagger}$ Zhi-Feng Li, ${ }^{*, \mathrm{~b}}$ Zhi-Jun Liu, ${ }^{\mathrm{a}}$ Edward F. Valeev, ${ }^{\mathrm{d}}$ \\ Lyudmila V. Moskaleva*,b,c, +
}

${ }^{a}$ School of Materials Science and Engineering, Jiangxi University of Science and Technology, Ganzhou 341000, People's Republic of China

${ }^{\mathrm{b}}$ Institute of Applied and Physical Chemistry and Center for Environmental Research and Sustainable Technology, University of Bremen, Bremen 28359,, Germany

${ }^{c}$ Department of Chemistry, University of the Free State, PO Box 339, Bloemfontein, South Africa

d Department of Chemistry Virginia Tech Blacksburg, Virginia 24061, USA

$\dagger$ These authors contributed equally to this work.

*Corresponding author's e-mail addresses: liyong@uni-bremen.de, jxlzfeng@163.com, lyudmila.moskaleva@gmail.com

$\$$ Present address: Department of Chemistry, University of the Free State, Bloemfontein 9300, South Africa 


\section{Experimental}

\subsection{Chemicals and materials}

Pyridine-3,5-dicarboxylic acid $\left(\mathrm{H}_{2} \mathrm{PDA}\right)$ and oxalic acid were purchased from Sinopharm group chemical reagents co., Ltd. (China). $\mathrm{Tb}\left(\mathrm{NO}_{3}\right)_{3}$ (99.99\%) was purchased from Ganzhou Xinzheng rare earth new material co., Ltd. All chemicals and solvents were analytical grade reagents and were used directly without further purification. Deionized water was used throughout.

\subsection{Synthesis of $\left\{\left[\mathrm{Tb}_{2}(\mathrm{PDA})_{2}(\mathrm{ox})\left(\mathrm{H}_{2} \mathrm{O}\right)_{4}\right]\left(\mathrm{H}_{2} \mathrm{O}\right)_{2}\right\}_{\mathrm{n}}$}

A mixture of $\mathrm{Tb}\left(\mathrm{NO}_{3}\right)_{3} \cdot 6 \mathrm{H}_{2} \mathrm{O}(0.5 \mathrm{mmol}, 0.2265 \mathrm{~g}), \mathrm{H}_{2} \mathrm{PDA}(0.5 \mathrm{mmol}, 0.0836 \mathrm{~g})$ and oxalic acid $(0.5 \mathrm{mmol}, 0.063 \mathrm{~g})$ dissolved in $14 \mathrm{~mL}$ mixture of water and methanol (v:v=1:1) was placed in a $25 \mathrm{~mL}$ Teflon-lined stainless steel autoclave. The autoclave was sealed and heated to $170{ }^{\circ} \mathrm{C}$ in an oven under autogenous pressure for $72 \mathrm{~h}$ and then cooled to room temperature. The colorless crystalline product was filtered, washed with ethanol and then dried at ambient temperature. The compound is stable and insoluble in water and most organic solvents.

\subsection{X-ray Structure Determination and Structure Refinement}

Diffraction data of the complex was collected using a Bruker Smart Apex II CCD with graphite monochromatic Mo $K_{\mathrm{a}}$ radiation source $(\lambda=0.71073 \AA)$ in $\omega$-scan technique at $296(2) \mathrm{K}$. The crystal structures were solved using direct methods and refined by full-matrix least squares on $\mathrm{F}^{2}$ using the SHELXL-97 package.[1] All hydrogen atoms were geometrically placed and refined in riding model approximation. The details of the crystal parameters, data collection and refinements for the complexes are summarized in Table S1. Selected bond lengths and angles are listed in Table $\mathrm{S} 2$.

\subsection{Fluorescence properties}

The photoluminescence spectra were measured on a FLS980 spectrofluorometer equipped with a xenon lamp as the excitation source at room temperature. 
Table S1. Crystallographic data of the complex $\left\{\left[\mathrm{Tb}_{2}(\mathrm{PDA})_{2}(\mathrm{ox})\left(\mathrm{H}_{2} \mathrm{O}\right)_{4}\right]\left(\mathrm{H}_{2} \mathrm{O}\right)_{2}\right\}_{\mathrm{n}}$

\begin{tabular}{ll}
\hline Empirical formula & $\mathrm{C}_{20} \mathrm{H}_{14} \mathrm{~Tb}_{2} \mathrm{~N}_{2} \mathrm{O}_{18}$ \\
\hline Formula weight & $844.16 \mathrm{~g} / \mathrm{mol}$ \\
Temperature/ $\mathrm{K}$ & $296(2)$ \\
Crystal System & Monoclinic \\
Space group & $\mathrm{P} 2{ }_{1} / \mathrm{n}$ \\
Unit cell parameters & \\
$\mathrm{a} / \AA$ & $\mathrm{a}=7.6331(4)$ \\
$\mathrm{b} / \AA$ & $\mathrm{b}=9.8362(5)$ \\
$\mathrm{c} / \AA$ & $\mathrm{c}=14.7770(8)$ \\
$\beta /$ degree & $\beta=98.303(1)$ \\
$\mathrm{Z}$ & 2 \\
Volume/ nm & \\
Density $($ calculated $) /\left(\mathrm{g} \cdot \mathrm{mm}^{-3}\right)$ & $1.0978(1)$ \\
Absorption coefficient $/ \mathrm{mm}^{-1}$ & 1.277 \\
$\mathrm{~F}(000)$ & 3.245 \\
Crystal size ( mm $\left.{ }^{3}\right)$ & 402 \\
$\theta /$ degree & $0.12 \times 015 \times 034$ \\
Limiting indices & 2.50 to 27.79 \\
Collected unique reflections & $-9 \leq \mathrm{h} \leq 9 ;-12 \leq \mathrm{k} \leq 12 ;-14 \leq \mathrm{l} \leq 19$ \\
\hline
\end{tabular}

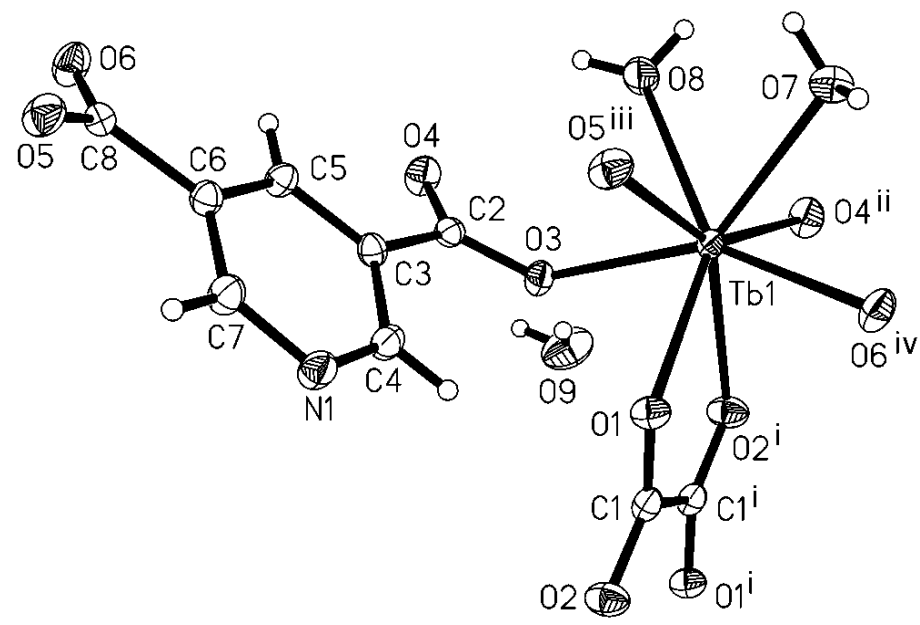

Figure S1. The asymmetric unit of the compound $\left\{\left[\mathrm{Tb}_{2}(\mathrm{PDA})_{2}(\mathrm{ox})\left(\mathrm{H}_{2} \mathrm{O}\right)_{4}\right]\left(\mathrm{H}_{2} \mathrm{O}\right)_{2}\right\}_{\mathrm{n}}$ (symmetry codes: $\mathrm{i}=1-\mathrm{x}, 2-\mathrm{y}, 1-\mathrm{z} ; \mathrm{ii}=0.5-\mathrm{x}, 0.5+\mathrm{y}, 0.5-\mathrm{-} ; \mathrm{iii}=-\mathrm{x}, 1-\mathrm{y}, 1-\mathrm{z} ; \mathrm{iv}=\mathrm{x}, 1+\mathrm{y}, \mathrm{z}$. ) 


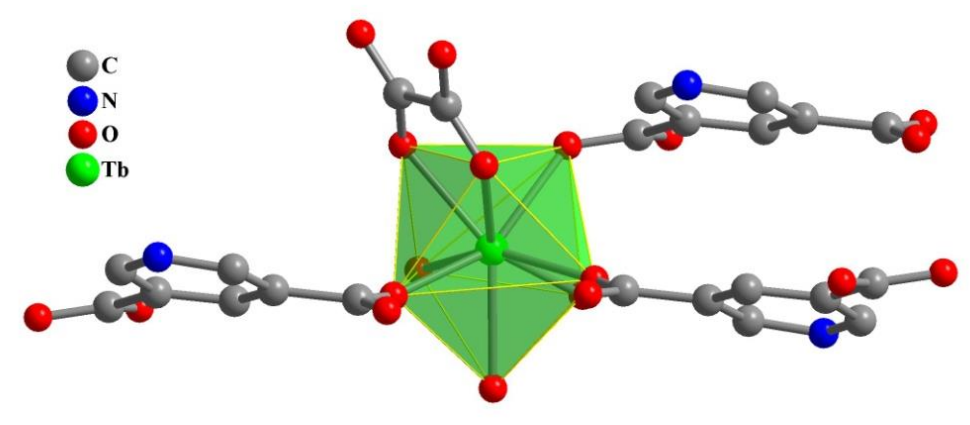

Figure S2. The coordination environment of $\mathrm{Tb}^{3+}$.

Table S2. Selected bond lengths $(\AA)$ for the compound $\left\{\left[\mathrm{Tb}_{2}(3,5-\mathrm{pdc})_{2}(\mathrm{ox})\left(\mathrm{H}_{2} \mathrm{O}\right)_{4}\right]\left(\mathrm{H}_{2} \mathrm{O}\right)_{2}\right\}$ n. Only the bond lengths of $\mathrm{Tb}-\mathrm{O}$ coordination bonds are listed. The remaining chemical bonds within organic ligands are not listed because the meta-hybrid functional is expected to predict them with reasonable accuracy. Additionally, the bond lengths of the simplified Model II computed using MP2 are listed.

\begin{tabular}{cccc}
\hline Bond length & Experimental & wB97XD (Model I) & MP2 (Model II) \\
Tb1-O1 & 2.47 & 2.41 & 2.30 \\
$\mathrm{~Tb} 1-\mathrm{O} 2$ & 2.43 & 2.39 & 2.31 \\
$\mathrm{~Tb} 1-\mathrm{O} 3$ & 2.35 & 2.33 & 2.41 \\
$\mathrm{~Tb} 1-\mathrm{O} 4$ & 2.33 & 2.37 & 2.42 \\
$\mathrm{~Tb} 1-\mathrm{O} 5$ & 2.30 & 2.36 & 2.41 \\
$\mathrm{~Tb} 1-\mathrm{O} 6$ & 2.30 & 2.35 & 2.41 \\
$\mathrm{~Tb} 1-\mathrm{O} 7$ & 2.45 & 2.55 & 2.55 \\
$\mathrm{~Tb} 1-\mathrm{O} 8$ & 2.45 & 2.54 & 2.54 \\
\hline
\end{tabular}




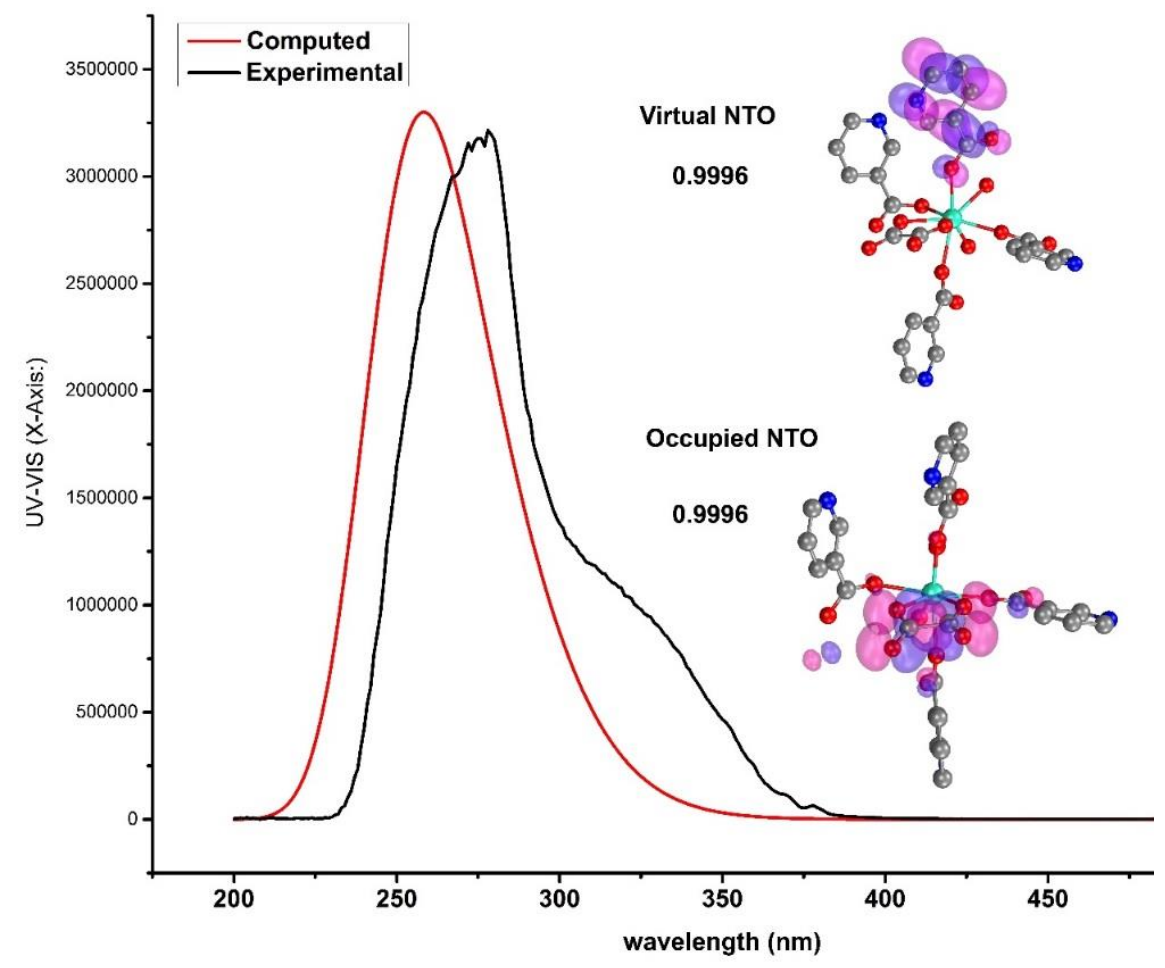

Figure S3. Experimental (black curve) and computed using the PBE0 functional (red curve) absorption spectra of the $\left\{\left[\mathrm{Tb}_{2}(\mathrm{PDA})_{2}(\mathrm{ox})\right]\left(\mathrm{H}_{2} \mathrm{O}\right)_{2}\right\}_{\mathrm{n}}$ complex. 

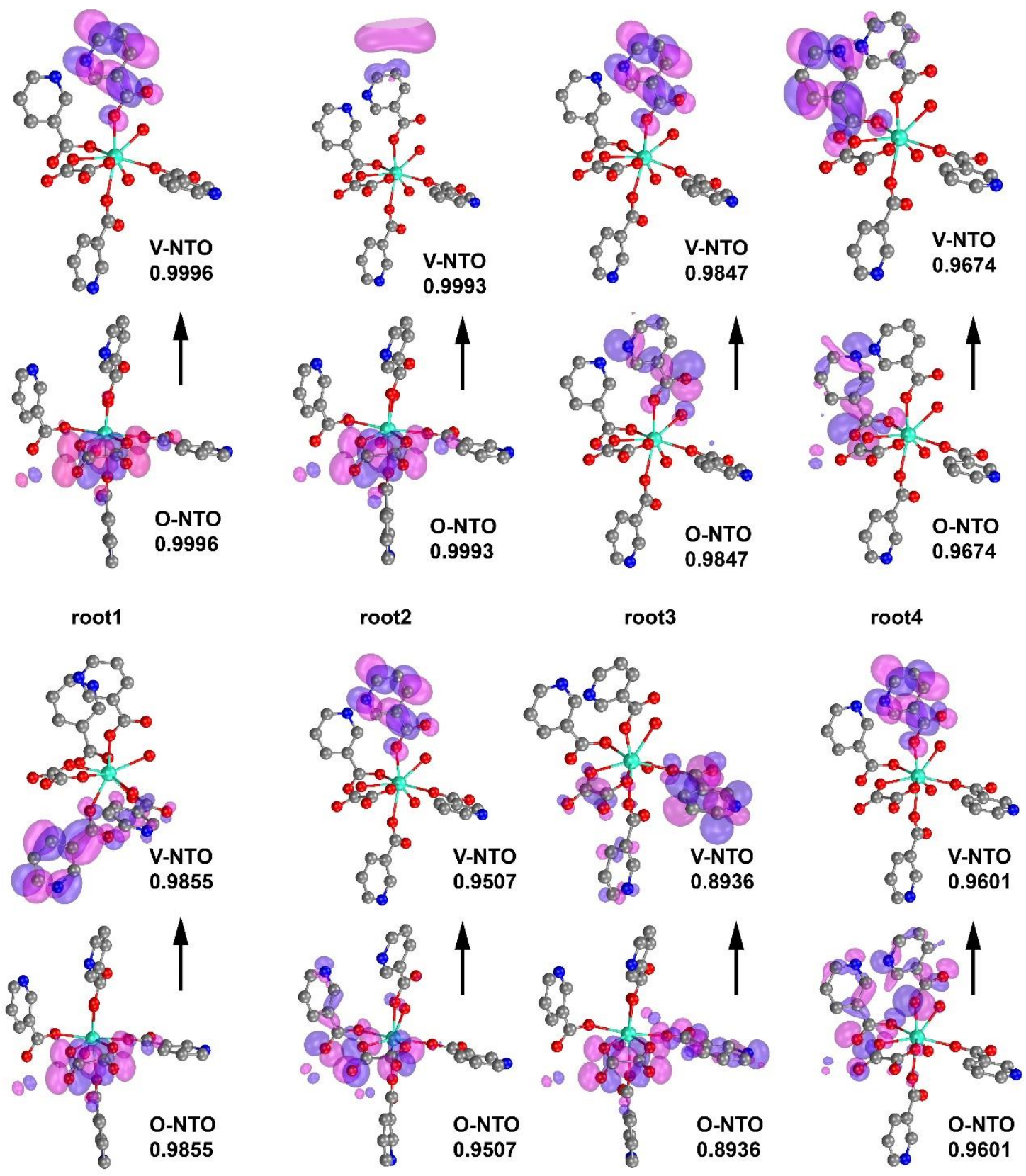

root5

root6

$\operatorname{root} 7$

root8

Figure S4. Natural transition orbitals of the first 8 excitations from root1 to root8. Orbital transitions mainly contributing to the excitations are listed. O-NTO and V-NTO stand for occupied and virtual NTOs, respectively. 
Table S3. Splitting of the spectral terms $\left(\right.$ in $\left.\mathrm{cm}^{-1}\right)$ of $\mathrm{Tb}^{\mathrm{III}}\left(4 \mathrm{f}^{8}\right)$ computed with the active space $(8,7)$. The energies of quintet terms in approach $a$ (footnote) are given relative to the ${ }^{5} D_{j, 1}$ term.

\begin{tabular}{|c|c|c|c|c|c|}
\hline Spectral Term & $J$ & CASSCF/SOC & CASSCF/SOC ${ }^{b}$ & CAS-NEVPT2/SOC & CAS-NEVPT2/SOC ${ }^{b}$ \\
\hline \multirow[t]{13}{*}{${ }^{7} \boldsymbol{F}$} & 6 & 0 & 0 & 0 & 0 \\
\hline & & 5.2 & 2.6 & 5.4 & 3.7 \\
\hline & & 38.7 & 53.4 & 42.2 & 51.0 \\
\hline & & 62.5 & 70.2 & 67.2 & 71.3 \\
\hline & & 97.7 & 111.3 & 106.0 & 115.8 \\
\hline & & 171.0 & 171.5 & 184.4 & 187.0 \\
\hline & & 174.4 & 174.3 & 187.9 & 188.5 \\
\hline & & 224.4 & 238.7 & 243.0 & 273.0 \\
\hline & & 225.1 & 239.9 & 243.7 & 274.1 \\
\hline & & 274.7 & 256.5 & 300.0 & 285.6 \\
\hline & & 275.0 & 258.6 & 300.2 & 287.2 \\
\hline & & 538.8 & 516.5 & 581.0 & 585.2 \\
\hline & & 538.8 & 516.5 & 581.0 & 585.3 \\
\hline \multirow[t]{11}{*}{${ }^{7} \boldsymbol{F}$} & 5 & 1778.2 & 1793.3 & 1783.2 & 1794.5 \\
\hline & & 1805.3 & 1816.4 & 1811.4 & 1817.6 \\
\hline & & 1844.3 & 1856.1 & 1857.2 & 1873.4 \\
\hline & & 1913.6 & 1921.4 & 1931.1 & 1944.6 \\
\hline & & 1932.5 & 1930.6 & 1946.9 & 1952.8 \\
\hline & & 1959.5 & 1960.7 & 1976.6 & 1987.0 \\
\hline & & 1982.6 & 1980.5 & 2001.0 & 2014.2 \\
\hline & & 2015.4 & 2012.8 & 2037.1 & 2044.7 \\
\hline & & 2018.3 & 2015.5 & 2041.0 & 2050.3 \\
\hline & & 2137.0 & 2119.9 & 2168.9 & 2171.5 \\
\hline & & 2144.2 & 2126.2 & 2177.5 & 2180.3 \\
\hline \multirow[t]{10}{*}{${ }^{7} \boldsymbol{F}$} & 4 & & & & \\
\hline & & 2960.3 & 3283.7 & 2936.4 & 3285.4 \\
\hline & & 2997.5 & 3313.0 & 2981.8 & 3325.8 \\
\hline & & 3045.2 & 3346.4 & 3027.8 & 3364.0 \\
\hline & & 3049.9 & 3349.5 & 3033.1 & 3367.7 \\
\hline & & 3132.4 & 3416.1 & 3124.9 & 3444.4 \\
\hline & & 3154.5 & 3438.3 & 3147.7 & 3470.0 \\
\hline & & 3181.4 & 3460.1 & 3171.4 & 3494.9 \\
\hline & & 3284.5 & 3544.4 & 3288.6 & 3597.4 \\
\hline & & 3330.5 & 3581.8 & 3336.2 & 3641.4 \\
\hline
\end{tabular}




\begin{tabular}{|c|c|c|c|c|c|}
\hline \multirow[t]{7}{*}{${ }^{7} F$} & 3 & 3889.4 & 4501.0 & 3849.3 & 4513.1 \\
\hline & & 3936.2 & 4536.4 & 3903.3 & 4558.3 \\
\hline & & 3945.4 & 4544.6 & 3909.8 & 4564.7 \\
\hline & & 3993.3 & 4569.7 & 3966.9 & 4598.7 \\
\hline & & 4005.3 & 4603.1 & 3979.4 & 4629.1 \\
\hline & & 4082.7 & 4646.2 & 4066.4 & 4691.2 \\
\hline & & 4102.9 & 4652.1 & 4088.8 & 4700.2 \\
\hline \multirow[t]{5}{*}{${ }^{7} \boldsymbol{F}$} & 2 & 4534.2 & 5358.6 & 4490.1 & 5380.4 \\
\hline & & 4608.5 & 5410.9 & 4575.4 & 5439.0 \\
\hline & & 4656.2 & 5468.5 & 4628.4 & 5505.1 \\
\hline & & 4712.0 & 5500.2 & 4685.4 & 5539.2 \\
\hline & & 4812.8 & 5609.8 & 4786.8 & 5662.4 \\
\hline \multirow[t]{3}{*}{${ }^{7} F$} & 1 & 4981.7 & 5947.7 & 4939.4 & 5972.3 \\
\hline & & 5066.5 & 6038.4 & 5030.2 & 6074.3 \\
\hline & & 5231.4 & 6205.6 & 5204.6 & 6261.2 \\
\hline${ }^{7} F$ & 0 & 5320.2 & 6371.2 & 5285.3 & 6414.0 \\
\hline \multirow[t]{9}{*}{${ }^{5} D$} & 4 & 0 & 0 & 0 & 0 \\
\hline & & 6.8 & 5.3 & 6.6 & 3.9 \\
\hline & & 31.3 & 21.6 & 36.5 & 33.1 \\
\hline & & 51.11 & 33.2 & 58.1 & 46.9 \\
\hline & & 55.8 & 41.1 & 65.2 & 65.6 \\
\hline & & 76.3 & 51.6 & 90.9 & 73.5 \\
\hline & & 78.9 & 56.1 & 94.9 & 76.6 \\
\hline & & 118.8 & 89.4 & 142.9 & 129.6 \\
\hline & & 119.0 & 90.5 & 143.5 & 130.3 \\
\hline \multirow[t]{7}{*}{${ }^{5} D$} & 3 & 5474.4 & 5152.2 & 5501.1 & 5166.6 \\
\hline & & 5475.3 & 5153.7 & 5504.1 & 5168.0 \\
\hline & & 5479.4 & 5154.0 & 5506.3 & 5173.9 \\
\hline & & 5479.5 & 5158.2 & 5509.3 & 5178.2 \\
\hline & & 5487.2 & 5159.2 & 5516.9 & 5178.3 \\
\hline & & 5487.3 & 5161.3 & 5521.0 & 5184.5 \\
\hline & & 5488.9 & 5161.6 & 5522.0 & 5184.7 \\
\hline \multirow[t]{4}{*}{${ }^{5} G$} & 4 & 6824.9 & 6950.1 & 6553.4 & 6641.0 \\
\hline & & 6828.7 & 6955.9 & 6557.6 & 6641.1 \\
\hline & & 6829.1 & 6959.5 & 6558.4 & 6669.0 \\
\hline & & 6831.3 & 6961.3 & 6561.3 & 6671.4 \\
\hline
\end{tabular}




\begin{tabular}{|c|c|c|c|c|c|}
\hline & & 6844.0 & 6971.5 & 6576.0 & 6684.5 \\
\hline & & 6854.4 & 6982.4 & 6588.3 & 6697.6 \\
\hline & & 6914.7 & 7042.9 & 6654.7 & 6765.1 \\
\hline & & 6940.9 & 7069.5 & 6685.6 & 6795.9 \\
\hline & & 6951.9 & 7084.7 & 6699.9 & 6815.5 \\
\hline \multirow[t]{7}{*}{${ }^{5} G$} & 3 & 7114.7 & 7249.2 & 6894.7 & 7008.6 \\
\hline & & 7218.6 & 7360.2 & 7007.8 & 7126.9 \\
\hline & & 7218.6 & 8064.0 & 7007.8 & 7126.9 \\
\hline & & 7715.1 & 8072.9 & 7351.3 & 7781.2 \\
\hline & & 7723.3 & 8076.9 & 7360.8 & 7790.6 \\
\hline & & 7727.4 & 8093.6 & 7361.4 & 7795.5 \\
\hline & & 7742.6 & 8118.5 & 7374.0 & 7813.9 \\
\hline \multirow[t]{5}{*}{${ }^{5} D$} & 2 & 7759.3 & 8120.5 & 7385.2 & 7838.3 \\
\hline & & 7761.5 & 8137.6 & 7387.5 & 7840.9 \\
\hline & & 7784.3 & 8165.4 & 7403.0 & 7863.4 \\
\hline & & 7811.2 & 8175.4 & 7405.1 & 7897.1 \\
\hline & & 7819.3 & 8202.0 & 7405.6 & 7904.6 \\
\hline \multirow[t]{5}{*}{${ }^{5} G$} & 2 & 7842.7 & 8203.6 & 7425.2 & 7936.1 \\
\hline & & 7844.1 & 8242.1 & 7427.3 & 7938.1 \\
\hline & & 7881.3 & 8243.3 & 7432.3 & 7983.7 \\
\hline & & 7883.1 & 8271.6 & 7454.3 & 7984.4 \\
\hline & & 7910.5 & 8276.1 & 7455.2 & 8016.8 \\
\hline \multirow[t]{3}{*}{${ }^{5} G$} & 1 & 7914.4 & 8294.4 & 7476.6 & 8025.7 \\
\hline & & 7933.6 & 8298.3 & 7480.8 & 8045.4 \\
\hline & & 7936.2 & 8403 & 7486.2 & 8054.1 \\
\hline \multirow[t]{3}{*}{${ }^{5} D$} & 1 & 8035.0 & 8974.1 & 7503.5 & 8163.9 \\
\hline & & 8620.5 & 8986.9 & 7509.0 & 8218.6 \\
\hline & & 8630.0 & 8998.7 & 7518.3 & 8222.8 \\
\hline${ }^{5} D$ & 0 & 8634.6 & 9010.7 & 7520.6 & 8310.3 \\
\hline
\end{tabular}

a. Including quintet and septet states.

b. Including only quintet or only septet states. 
Table S4. The emission energies from ${ }^{5} D_{4} \rightarrow{ }^{7} F_{\mathrm{J}}$, calculated as $E\left({ }^{5} D_{4}\right)-E\left({ }^{7} F_{\mathrm{J}}\right)$ compared to experimental values.

\begin{tabular}{|c|c|c|c|}
\hline \multirow[t]{2}{*}{ Transition } & \multicolumn{2}{|c|}{ Transition Energy (nm) } & \multirow[b]{2}{*}{ Experimental } \\
\hline & CAS-NEVPT2 ${ }^{\text {a }}$ & CAS-NEVPT $2^{b}$ & \\
\hline \multirow[t]{13}{*}{${ }^{5} D_{4} \rightarrow{ }^{7} F_{6}$} & 464.9 & 485.11 & \\
\hline & 465.0 & 485.20 & \\
\hline & 465.8 & 486.31 & \\
\hline & 466.4 & 486.78 & \\
\hline & 467.2 & 487.86 & 486 to 496 \\
\hline & 468.9 & 489.55 & \\
\hline & 469.0 & 489.59 & \\
\hline & 470.2 & 491.61 & \\
\hline & 470.2 & 491.65 & \\
\hline & 471.5 & 491.92 & \\
\hline & 471.5 & 491.96 & \\
\hline & 477.8 & 499.29 & \\
\hline & 477.8 & 499.29 & \\
\hline \multirow[t]{11}{*}{${ }^{5} D_{4} \rightarrow{ }^{7} F_{5}$} & 506.9 & 531.37 & \\
\hline & 507.7 & 532.03 & \\
\hline & 508.9 & 533.61 & \\
\hline & 510.8 & 535.63 & \\
\hline & 511.2 & 535.87 & 540 to 552 \\
\hline & 512.0 & 536.86 & \\
\hline & 512.6 & 537.63 & \\
\hline & 513.6 & 538.52 & \\
\hline & 513.7 & 538.68 & \\
\hline & 517.1 & 542.22 & \\
\hline & 517.3 & 542.48 & \\
\hline \multirow[t]{9}{*}{${ }^{5} D_{4} \rightarrow{ }^{7} F_{4}$} & 538.4 & 577.07 & \\
\hline & 539.7 & 578.42 & \\
\hline & 541.1 & 579.71 & \\
\hline & 541.3 & 579.82 & 581 to 590 \\
\hline & 543.9 & 582.44 & \\
\hline & 544.6 & 583.29 & \\
\hline & 545.3 & 584.14 & \\
\hline & 548.8 & 587.65 & \\
\hline & 550.3 & 589.19 & \\
\hline${ }^{5} D_{4} \rightarrow{ }^{7} F_{3}$ & 566.3 & 621.10 & \\
\hline
\end{tabular}




\begin{tabular}{|c|c|c|c|}
\hline & 568.0 & 622.84 & \\
\hline & 568.2 & 623.09 & \\
\hline & 570.0 & 624.41 & 617 to 623 \\
\hline & 570.5 & 625.58 & \\
\hline & 573.3 & 628.02 & \\
\hline & 574.0 & 628.40 & \\
\hline${ }^{5} D_{4} \rightarrow{ }^{7} F_{2}$ & 587.6 & 656.45 & \\
\hline & 590.5 & 658.99 & 647 to 650 \\
\hline & 592.4 & 661.84 & \\
\hline & 594.4 & 663.37 & \\
\hline & 598.0 & 668.81 & \\
\hline${ }^{5} D_{4} \rightarrow{ }^{7} F_{I}$ & 603.5 & 682.99 & \\
\hline & 606.8 & 687.76 & 679 to 680 \\
\hline & 613.3 & 696.73 & \\
\hline${ }^{5} D_{4} \rightarrow{ }^{7} F_{0}$ & 616.4 & 704.21 & \\
\hline
\end{tabular}

a. Approach $a$ : including both quintet and septet states.

b. Approach $b$ : including only quintet or only septet states.

Table S5. Comparison of different computational approaches. The transition energies were computed and compared at the scalar relativistic and spin-orbit coupling level. The influence of including diffuse functions in the basis sets of light elements on transition energies is also considered.

\begin{tabular}{ccccccc}
\hline \multirow{2}{*}{ Transition } & \multicolumn{3}{c}{ Scalar relativistic } & & \multicolumn{2}{c}{ Spin-orbit coupling } \\
\cline { 2 - 3 } & CAS-NEVPT2 $^{\mathrm{a}}$ & CAS-NEVPT2 $^{\mathrm{b}}$ & CAS-NEVPT2 $^{\mathrm{b}, \mathrm{c}}$ & & CAS- NEVPT2/SOC $^{\mathrm{b}}$ & CAS-NEVPT2/SOC $^{\mathrm{a}}$ \\
${ }^{\mathbf{5}} \boldsymbol{D}_{\boldsymbol{4}} \rightarrow{ }^{\boldsymbol{7}} \boldsymbol{F}_{\boldsymbol{6}}$ & 2.9510 & 2.8743 & 2.8640 & & 2.5558 & 2.6668 \\
\hline
\end{tabular}

CAS- NEVPT2 calculations included quintet and septet states (approach $a$ ).

a. CAS- NEVPT2 calculations included either only quintet or only septet states (approach $b$ ).

b. Basis sets of light elements did not include diffuse functions. 
Table S6. The effect of including diffuse functions in the basis sets of light elements on excitation energies.

\begin{tabular}{|c|c|c|c|c|}
\hline Spectral Term & ${ }^{\mathrm{a} C A S S C F} / \mathrm{SOC}$ & ${ }^{\mathrm{b}} \mathrm{CASSCF} / \mathrm{SOC}$ & ${ }^{\mathrm{a} C A S-N E V P T 2 / S O C}$ & ${ }^{\mathrm{b}} \mathrm{CAS}-\mathrm{NEVPT} 2 / \mathrm{SOC}$ \\
\hline \multirow[t]{13}{*}{${ }^{7} F_{6}$} & 0.0 & 0.0 & 0.0 & 0.0 \\
\hline & 2.6 & 2.6 & 3.8 & 3.7 \\
\hline & 52.7 & 53.4 & 50.7 & 50.9 \\
\hline & 69.8 & 70.2 & 71.6 & 71.3 \\
\hline & 109.8 & 111.3 & 115.1 & 115.8 \\
\hline & 170.5 & 171.5 & 187.1 & 187.0 \\
\hline & 172.6 & 174.3 & 188.1 & 188.5 \\
\hline & 236.6 & 238.7 & 273.8 & 273.0 \\
\hline & 237.7 & 239.9 & 275.1 & 274.1 \\
\hline & 256.0 & 256.6 & 284.8 & 285.6 \\
\hline & 258.0 & 258.6 & 286.8 & 287.2 \\
\hline & 518.6 & 516.5 & 588.2 & 585.2 \\
\hline & 518.6 & 516.5 & 588.3 & 585.3 \\
\hline \multirow[t]{11}{*}{${ }^{7} F_{5}$} & 1792.2 & 1793.3 & 1794.1 & 1794.5 \\
\hline & 1815.5 & 1816.4 & 1817.7 & 1817.6 \\
\hline & 1854.6 & 1856.1 & 1872.7 & 1873.4 \\
\hline & 1920.4 & 1921.4 & 1944.9 & 1944.6 \\
\hline & 1929.3 & 1930.6 & 1952.7 & 1952.8 \\
\hline & 1960.3 & 1960.7 & 1987.5 & 1987.0 \\
\hline & 1980.2 & 1980.5 & 2014.7 & 2014.2 \\
\hline & 2013.2 & 2012.8 & 2045.5 & 2044.7 \\
\hline & 2015.9 & 2015.5 & 2051.2 & 2050.3 \\
\hline & 2120.0 & 2119.9 & 2172.8 & 2171.5 \\
\hline & 2126.2 & 2126.2 & 2181.5 & 2180.3 \\
\hline \multirow[t]{9}{*}{${ }^{7} F_{4}$} & 3283.0 & 3283.7 & 3286.1 & 3285.4 \\
\hline & 3312.4 & 3313.0 & 3326.7 & 3325.8 \\
\hline & 3345.9 & 3346.4 & 3363.6 & 3364.0 \\
\hline & 3349.0 & 3349.5 & 3368.0 & 3367.7 \\
\hline & 3415.8 & 3416.1 & 3445.1 & 3444.4 \\
\hline & 3438.0 & 3438.3 & 3470.6 & 3469.9 \\
\hline & 3459.3 & 3460.1 & 3494.3 & 3494.9 \\
\hline & 3544.0 & 3544.4 & 3598.1 & 3597.4 \\
\hline & 3581.3 & 3581.8 & 3642.3 & 3641.4 \\
\hline \multirow[t]{3}{*}{${ }^{7} F_{3}$} & 4500.7 & 4501.1 & 4513.4 & 4513.1 \\
\hline & 4536.1 & 4536.4 & 4559.1 & 4558.3 \\
\hline & 4544.2 & 4544.6 & 4565.1 & 4564.7 \\
\hline
\end{tabular}




$\begin{array}{ccccc} & 4569.2 & 4569.7 & 4599.0 & 4598.7 \\ & 4602.1 & 4603.2 & 4629.4 & 4629.1 \\ & 4645.4 & 4646.2 & 4691.5 & 4691.2 \\ & 4651.8 & 4652.1 & 4700.9 & 4700.2 \\ { }^{7} \boldsymbol{F}_{\mathbf{2}} & & & \\ & & & 5380.4 & 5380.4 \\ & 5357.9 & 5358.6 & 5439.9 & 5439.0 \\ & 5410.7 & 5410.9 & 5505.4 & 5505.1 \\ & 5467.6 & 5468.5 & 5539.8 & 5539.2 \\ & 5499.7 & 5500.2 & 5662.3 & 5662.4 \\ & 5608.8 & 5609.8 & & 5972.3 \\ { }^{7} \boldsymbol{F}_{\mathbf{1}} & & & 5973.1 & 6074.3 \\ & 5947.8 & 5947.7 & 6073.5 & 6261.2 \\ & 6036.3 & 6038.4 & 6263.6 & 6413.9\end{array}$

a. Excitation energies calculated without including diffuse functions on light elements.

b. Excitation energies calculated including diffuse functions on light elements.

\section{References}

[1] G. M. Sheldrick, SHELXL-97. Program for the Refinement of Crystal Structures. University of Göttingen, Germany 1997. 